\title{
Fabrication and characterization of poly(octanediol citrate)/ gallium-containing bioglass microcomposite scaffolds
}

\author{
Ehsan Zeimaran - Sara Pourshahrestani - Belinda Pingguan-Murphy • \\ Nahrizul Adib Kadri · Hussin A. Rothan · Rohana Yusof • \\ Mark R. Towler · Ivan Djordjevic
}

\begin{abstract}
Bone can be affected by osteosarcomae requiring surgical excision of the tumor as part of the treatment regime. Complete removal of cancerous cells is difficult and conventionally requires the removal of a margin of safety around the tumor to offer improved patient prognosis. This work considers a novel series of composite scaffolds based on poly(octanediol citrate) (POC) impregnated with galliumbased bioglass microparticles for possible incorporation into bone following tumor removal. The objective of this research was to fabricate and characterize these scaffolds and subsequently report on their mechanical and biological properties. The porous microcomposite scaffolds with various concentrations of bioglass $(10,20,30 \mathrm{wt} \%)$ incorporated were fabricated using a salt leaching technique. The scaffolds exhibited compression modulus in the range of 0.3-7 MPa. The addition of bioglass increased the mechanical properties even though porosity increased. Furthermore, increasing the concentration of bioglass had a significant influence on glass transition temperature from $2.5{ }^{\circ} \mathrm{C}$ for the pure polymer to around $25^{\circ} \mathrm{C}$ for $30 \%$ bioglass-containing composite. The ion release study revealed that composites containing $10 \%$ bioglass had the highest ion release ratio after 28 days of soaking in
\end{abstract}

E. Zeimaran - S. Pourshahrestani - B. Pingguan-Murphy

N. A. Kadri · M. R. Towler · I. Djordjevic (夰)

Department of Biomedical Engineering, Faculty of Engineering, University of Malaya, 50603 Kuala Lumpur, Malaysia

e-mail: ivandjordjevich@hotmail.com

H. A. Rothan · R. Yusof

Department of Molecular Medicine, Faculty of Medicine,

University of Malaya, 50603 Kuala Lumpur, Malaysia

M. R. Towler

Department of Mechanical \& Industrial Engineering, Ryerson

University, Toronto, ON M5B 2K3, Canada phosphate buffered saline. The interaction of bioglass phase with POC led to the formation of additional ionic crosslinks aside from covalent crosslinks which further resulted in increased stiffness and decreased weight loss. The osteoblast cells were well attached and growth on composites and collagen synthesis increased particularly with the $10 \%$ bioglass concentration.

\section{Introduction}

Bone defects caused by tumor reconstruction, chronic infection, or traumatic bone loss create a major surgical problem [1]. The majority of fractures heal well under standard conservative or surgical therapy. However, extended bone defects following trauma or cancer resection or non-unions of fractures may require more sophisticated treatments [2]. The accepted clinical standard for bone defect treatment and nonunion is autologous bone grafting. This treatment presents serious problems with donor site morbidity, prolonged operation time, and the limited availability of graft materials [3]. Allograft bone also has concerns related to disease transmission risk and infection, explaining why synthetic bone grafts are increasingly being employed in the clinical field [1]. Certain biomaterials can repair or replace damaged or diseased tissue by mimicking the natural extracellular matrix (ECM) [4]. Bio-resorbable scaffolds degrade in situ and minimize the need for additional surgery to remove the implant [5]. An ideal scaffold should degrade at a rate compatible with the rate of bone growth, physically creating open space for new bone formation until full regeneration is achieved. From a mechanical viewpoint, an ideal bone scaffold must provide sufficient mechanical support for preserving tissue volume and consequently promotion of tissue regeneration [6]. 
Bioactive glasses are increasingly employed for bone void filling. The first commercially available bioactive glass, known as "45S5", has shown positive interaction with both hard and soft tissues [7]. However, the lack of versatility in material processing and inherent brittleness of bioactive glasses limits their applicability in load bearing applications $[8,9]$. Previous research postulates that polymer/bioglass composite materials could combine the osteoconductive properties, stiffness and strength of bioactive glasses with the processability of biodegradable polymers in order to increase the applicability of glass-based materials for tissue augmentation $[10,11]$.

Gallium $(\mathrm{Ga})$ is a known chemotherapeutic ion and its biological function has been approved by food and drug administration (FDA) $[12,13]$. It is also accepted that Ga prevents osteoclast bone resorption by increasing calcium (Ca) concentration without influencing osteoblasts [14]. Bioglass materials containing $\mathrm{Ga}$ have applicability for treatment of bone cancer due to several reasons, as Ga ions: (1) restrict osteoclast activity in bone without changing viability of these cells; (2) decrease crystal solubility in the physiological environment; and (3) increase bone Ca content resulting in less bone resorption.

The anti-inflammatory and immunosuppressive Ga-compounds have also been proven to work in animal models of human diseases $[12,15]$. In order to utilize the potential of Ga-containing bioglass, they need to be delivered to the body in scaffolds with a controlled release dosage form. Composite materials including biodegradable polyesters are possible candidates for bioactive porous scaffolds.

Biodegradable polyester elastomeric materials based on citric acid (CA) have been used in various tissue engineering applications as prominent new scaffolds with optimal physico-chemical properties [16, 17]. In particular, a polyester thermoset elastomer, polyoctanediol citrate (POC) is a polymer of interest in this work due to proven biomimetic properties, tunable degradation rate, and synthesis/processing procedures that can be repeated in standard laboratory conditions [18]. Composites of POC with hydroxyapatite (HA) have been considered for bone replacement and augmentation $[19,20]$. So far, no attempt has been made to produce Ga-containing bioglass composites with biocompatible POC elastomers.

The major aim of the current study is to produce a bone tissue engineering scaffold which has the ability to release therapeutic ions from a $\mathrm{Si}-\mathrm{Ca}-\mathrm{Zn}-\mathrm{Ga}$ bioglass phase, offering a better clinical outcome particularly for sufferers of osteosarcomae. We have fabricated porous scaffolds with different quantities of bioglass by solvent-casting/ particulate-leaching technique. The POC/bioglass scaffolds were characterized for their chemical structure, composite morphology and in vitro biodegradation. The ion-release kinetics was evaluated in detail in order to establish a novel composite scaffold with controlled release properties. The human-derived osteoblast cells were seeded on POC/bioglass scaffolds and results reveal a significant potential for both in vitro bone tissue engineering and future clinical applications in vivo.

\section{Experimental}

Materials

High purity CA, 1, 8-octanediol (OD), 1, 4-dioxane, and phosphate buffer saline (PBS, pH 7.4) pellets were used for scaffold processing (Sigma-Aldrich, $\geq 98 \%$ ). The analytical reagent of silica, calcium oxide, zinc oxide, and gallium oxide were used for glass synthesis (Sigma-Aldrich, $\geq 98 \%$ ).

Synthesis of bioglass

Bioglass with the formulation $0.48 \mathrm{SiO}_{2}-0.12 \mathrm{CaO}-$ $0.32 \mathrm{ZnO}-0.08 \mathrm{Ga}_{2} \mathrm{O}_{3}$ (representing molar fractions) was synthesized through a conventional melt-quench method. Briefly, the proper amounts of reagents were weighed out and ball milled for $1 \mathrm{~h}$. The mixture was dried and fired at $1500{ }^{\circ} \mathrm{C} 1 \mathrm{~h}$ in a platinum crucible and then quenched into water. The obtained frit was then ground using a ball mill and sieved to a particle size below $45 \mu \mathrm{m}$ [15].

\section{Fabrication of POC/bioglass films}

POC pre-polymer was synthesized by a polycondensation reaction reported elsewhere $[21,22]$. Briefly, equimolar amounts of $\mathrm{CA}$ and $\mathrm{OD}$ were weighed out and reacted in elevated temperature $\left(140{ }^{\circ} \mathrm{C}\right)$ for $1 \mathrm{~h}$ under nitrogen gas in order to produce $\mathrm{POC}$ pre-polymer. The POC/bioglass composite films were prepared through a solvent-casting technique. The predetermined amount of POC pre-polymer was dissolved in dioxane to obtain a $20 \%$ solution. Three different concentrations of bioglass (i.e., 10, 20 and $30 \% \sim$ w/w to pure POC pre-polymer) were added to the solution and sonicated (Ultrasonic, ST-UB5200LT, SASTEC) for $30 \mathrm{~min}$. The composites were named POC-BG$10 \%$ (10\% bioglass), POC-BG-20\% (20\% bioglass) and POC-BG-30 \% (30\% bioglass). The mixtures were then transferred into a Teflon ${ }^{\mathrm{TM}}$ casts with $7 \mathrm{~mm}$ diameter and left in oven at $80^{\circ} \mathrm{C}$ for 7 days. The resultant films with approximately $1 \mathrm{~mm}$ thickness were taken out from the mould and cut to the cylindrical shapes for characterization.

Fabrication of POC/bioglass scaffolds

Composite scaffolds were prepared by employing a saltleaching method. First, the POC pre-polymer was dissolved 
in dioxane in a polyethylene container to obtain a $20 \%$ solution. Then different concentrations of bioglass (10, 20 and $30 \mathrm{wt} \%$ ) were added to the solution and sonicated for 30 min. After that, $90 \%$ of sieved salt $(\mathrm{NaCl})$ was added to the mixture (w/w to solvent-free POC-BG mixture) and left in the oven at $80^{\circ} \mathrm{C}$ for 1 week. After curing and solvent evaporation, the $\mathrm{NaCl}-\mathrm{POC}-\mathrm{BG}$ blocks were removed from the container and $\mathrm{NaCl}$ was washed for 4 days in distilled water. The water was replenished every $12 \mathrm{~h}$. Once the washing was completed, the resultant scaffolds were frozen at $-80^{\circ} \mathrm{C}$ and cut to the cylindrical shape $(6 \mathrm{~mm}$ diameter and $5 \mathrm{~mm}$ height). Finally, the scaffolds were lyophilized using a freeze-dryer (FreeZone 2.5, LABCONCO, $7670531)$

Characterization of scaffolds

Fourier transform infrared spectroscopy (FTIR)

Infra-red spectra of scaffolds were recorded in attenuated total reflectance mode (ATR-FTIR400, Perkin Elmer instruments, USA) within the wavelength range of $400-4000 \mathrm{~cm}^{-1}$ in room temperature.

Thermal analysis

The glass transition temperature $\left(T_{\mathrm{g}}\right)$ of scaffolds was measured by differential scanning calorimetry (DSC8000,

Perkin Elmer instruments, USA). A sample of $5-10 \mathrm{mg}$ was encapsulated in standard aluminum pans and all tests were performed under a nitrogen atmosphere. The samples were analyzed at a rate of $10{ }^{\circ} \mathrm{C} / \mathrm{min}$ between -50 and $150{ }^{\circ} \mathrm{C}$. The samples were first heated up to $150{ }^{\circ} \mathrm{C}$ (first heating cycle), then cooled down to $-50{ }^{\circ} \mathrm{C}$ and finally, heated up again to $150{ }^{\circ} \mathrm{C}$ (second heating cycle). $T_{\mathrm{g}}$ was measured from the DSC endothermic curves in the second heating cycle. The effect of temperature on the composite scaffolds was evaluated by thermogravimetric analysis (TGA4000, Perkin Elmer instruments, USA). $5 \mathrm{mg}$ samples were heated in air atmosphere from 35 to $900{ }^{\circ} \mathrm{C}$ at a heating rate of $10{ }^{\circ} \mathrm{C} / \mathrm{min}$ using aluminum crucible.

\section{Mechanical tests and porosity}

Dynamic mechanical analysis (DMA) was performed on prepared scaffolds. Rectangular samples with dimension of $35 \times 13 \times 2 \mathrm{~mm}$ (length $\times$ width $\times$ thickness) were analyzed using a DMA 8000 (Perkin Elmer instruments, USA). The data were collected at temperature scan with dual cantilever bending geometry. The samples were scanned in the temperature range of $-60{ }^{\circ} \mathrm{C}$ to $+40{ }^{\circ} \mathrm{C}$ at a heating rate of $2{ }^{\circ} \mathrm{C} / \mathrm{min}$, oscillation frequency of $1 \mathrm{~Hz}$ and maximum strain level of $0.05 \mathrm{~mm}$. Mechanical compression tests were conducted to determine compressive modulus. The cylindrical scaffolds were tested using an Instron 5544 (USA) mechanical tester fitted with a $2 \mathrm{KN}$ load cell at a crosshead speed of $1 \mathrm{~mm} / \mathrm{min}(n=6)$. The specimens were compressed to $50 \%$ of initial volume. The average compressive modulus $\left(E_{\mathrm{c}}\right)$ and standard deviation was calculated by slope of initial linear region of strain-stress curve. The recovery ratio of the specimens was measured after 1 min uploading the pressure. Porosity was measured by Archimedes principle [23].

Field emission scanning electron microscopy (FESEM)

Scaffold morphology was investigated by FESEM (ZeissAuriga laser, Germany). The samples were placed on aluminum stubs with $8 \mathrm{~mm}$ diameter and the images were taken in various magnifications and acceleration voltage of $15 \mathrm{kV}$.

In vitro degradation test

The weight loss of samples was determined by soaking scaffold cylinders in PBS for 7, 14, 21, 28 days. Samples were immersed in $10 \mathrm{ml}$ PBS in $15 \mathrm{ml}$ centrifuge tubes. The tubes were kept diagonal and moderately shaken at $37{ }^{\circ} \mathrm{C}$ using orbital shaker incubator (Benchtop, Ind. \& Vac. Instrument). The PBS was refreshed every week. The scaffolds were removed from PBS at the set time intervals,

washed three times with distilled water and oven dried to a constant weight. The weight loss was recorded $(\%)$ and plotted against time intervals $(n=5)$.

\section{Contact angle measurements}

Static contact angle was carried out on films to measure the hydrophilicity of the samples. The measurement was carried out at room temperature by a sessile drop method using a high-performance image processing system from Dataphysics Instruments (OCA 15EC, Germany). Samples were tested by adding equal volume of water $(2 \mu \mathrm{l})$ using motor driven syringe. Photos were recorded after $2 \mathrm{~min}$ and water contact angles were measured by analyzing the drop images $(n=5)$.

\section{Swelling studies}

The swelling experiment was performed on composite films with $1 \mathrm{~mm}$ thickness both in water and in PBS. The dry weight of the sample was noted as $M_{0}$. Both PBS and water experiments were performed in time intervals for $6 \mathrm{~h}$. After the predetermined time, the water was removed from the sample surface by gently dabbing onto a tissue paper and the new weight $\left(M_{\mathrm{w}}\right)$ was recorded $(n=5)$. The 
ratio of swelling was calculated using equation $\left[\left(M_{\mathrm{w}}-\right.\right.$ $\left.\left.M_{0}\right) / M_{0}\right] \times 100$.

\section{Ion release studies}

Ion release studies were carried out on cylindrical scaffold samples with the weight $11-14 \mathrm{mg}$ in $10 \mathrm{ml}$ PBS at $37{ }^{\circ} \mathrm{C}$ in an orbital shaker incubator $(n=3)$. At specified time points $(1,7,14,28$ days), the scaffolds were removed from the centrifuge tubes and the solution was kept for further analysis. The PBS solutions were characterized by microwave plasma-atomic emission spectrometer (MP-AES) Agilent 4100 (Agilent Technologies, Inc., Santa Clara, CA, USA) to determine the concentration of $\mathrm{Si}, \mathrm{Ca}, \mathrm{Zn}$ and $\mathrm{Ga}$ for each time period. The ion release ratio was measured using the fractional ion release $\left(M_{\mathrm{t}} / M_{\infty}\right)$ where, $M_{\mathrm{t}}$ is the mass of ion released at the specified time intervals and $M_{\infty}$ is the absolute bioglass mass in the composites [24].

Cell culture, attachment and morphology of osteoblasts

Human osteoblast cells were purchased from Lonza (Lonza, USA,cat. \#CC-2538) and expended in growth medium consisting of Dulbecco's modified Eagle's medium (DMEM; Gibco-Invitrogen, USA) supplemented with $20 \%$ fetal bovine serum (FBS; Gibco -Invitrogen, USA), $100 \mu \mathrm{M}$ 2-mercaptoethanol (Sigma-Aldrich, USA), $100 \mathrm{U} /$ $\mathrm{ml}$ penicillin and $100 \mu \mathrm{g} / \mathrm{ml}$ streptomycin (Gibco-Invitrogen, USA) and incubated at standard conditions (humidified atmosphere, $5 \% \mathrm{CO}_{2}, 37{ }^{\circ} \mathrm{C}$ ) for enough expansion.

Before seeding the cells into scaffolds, the scaffolds were placed in 24 well plates, washed three times with PBS, left to dry at room temperature and placed under UV for overnight. The sterilised scaffold was soaked with FBSfree DMEM for $3 \mathrm{~h}$, the media were removed and the scaffold was left for another $3 \mathrm{~h}$ in the incubator. Then, the desired amount of the cells were dropped into the scaffolds and left for another $1 \mathrm{~h}$ in the incubator $\left(37{ }^{\circ} \mathrm{C}\right.$ and $5 \%$ $\mathrm{CO}_{2}$ ). Then the cell-seeded scaffolds were transferred to new plates before they were topped up with the media.

Cells were cultured for 7 days into 3D culture system $\left(1 \times 10^{5} /\right.$ scaffold $)$ and then fixed with $3 \%$ glutaraldehyde in PBS for $24 \mathrm{~h}$ at $4{ }^{\circ} \mathrm{C}$. After thorough washing with PBS, scaffold samples were dehydrated sequentially in 50,70 , 95 , and $100 \%$ ethanol. Then the fixed samples were freeze-dried, sputter coated with gold, and observed under SEM (HitachiS-530).

\section{Real-time PCR analysis of osteoblasts}

The scaffolds were seeded by human osteoblast cells and the measurement was performed after 7 days culturing. Real-Time PCR (RT-PCR) was performed by ABI 7500 instrument using Syber green ${ }^{\mathrm{TM}}$ fluorescence dye (Applied Biosystems, USA.) to assess the expression of genes of interest. The primers were designed using ABI 7500 instrument software, SDS2.1 for studying collagen I expression (COLI-F-5-CCTGGATGCCATCAAAGTCT-3 and COLI-R-5- GAATCCATCGGTCATGCTCT-3) and collagen III expression (COLIII-F-5-CTAAAGGCGAAG ATGGCAAG-3 and COLIII-R-5-TTTCCCATCACTTCC TGGTC-3).

\section{Indirect immunostaining}

Cells were grown into the scaffolds $\left(5 \times 10^{5} /\right.$ scaffold $)$ for 7 days. Then, cells were trypsinised and reseeded on cover slides fixed in 6-well plates and for $24 \mathrm{~h}$. Next, the cells were washed three times with PBS and fixed with ice-cold methanol for $15 \mathrm{~min}$ at $-20^{\circ} \mathrm{C}$. After the washing steps, the cells were incubated with a coating buffer for $1 \mathrm{~h}$ at room temperature. A mouse antibody specific to collagen I (abcam, USA, cat. \# ab90395) or collagen III (abcam, USA, cat. \# ab3610) were add separately and the cells were incubated for overnight at $4{ }^{\circ} \mathrm{C}$. The cells were washed three times with PBS and incubated for $30 \mathrm{~min}$ with an anti-mouse IgG labeled with FITC fluorescent dye (Invitrogen, USA, cat. \# 62-6511).

\section{Results and discussion}

FTIR analysis

FTIR analysis was performed in order to characterize the type of interactions and crosslinking in both pure POC samples and the micro-composites. Figure 1 presents the FTIR spectra for composite scaffolds. The presence of ester bonds was confirmed by the two characteristic peaks at 1726 and $1175 \mathrm{~cm}^{-1}$ ascribed to $\mathrm{C}=\mathrm{O}$ and $\mathrm{C}-\mathrm{O}$ stretching respectively which indicates successful polymerization of POC [25]. The results indicated that ester bands are more intensified for pure $\mathrm{POC}$ in respect to composites. It is inferred that $\mathrm{Ca}$ ions released from the bioglass interacted with free carboxylic acid groups in the POC resulting in a new carboxylate peak at $1621 \mathrm{~cm}^{-1}$ [26]. Thus, the reaction between the bioglass and POC led to a decrease in ester formation due to the ionic interaction of metallic carboxylate. The presence of this peak confirmed the hydrolysis of bioglass by the unreacted acid functionalities in the pre-polymer and consequently resulted in reduction of ester formation.

Thermal analysis

The thermogravimetric traces from pure POC and its microcomposites are shown in Fig. 2. The results show that, 
Fig. 1 Representative ATR FTIR spectrum of POC-BG composite; insert showing expanded spectral region for: pure POC (black); POC-BG$10 \%$ (red); POC-BG-20\% (green); and POC-BG-30\% (blue) (Color figure online)

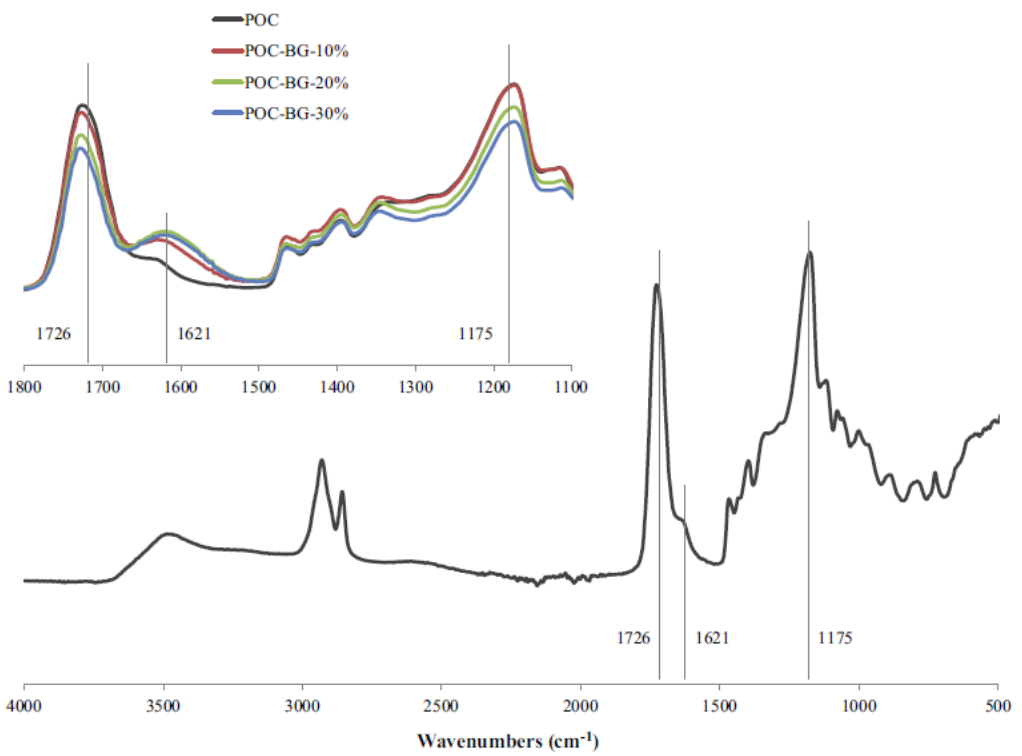

as a result of precipitation and agglomeration phenomena [27].

Data from DSC thermograms of the pure POC and composites are shown in Table 1. All samples were found to be amorphous at body temperature with $T_{\mathrm{g}}$ ranging from 2.5 to $25{ }^{\circ} \mathrm{C}$. In addition, $T_{\mathrm{g}}$ values increased as the amount of bioglass increased. This can be ascribed to the higher rate of carboxylate formation in respect to ester bonds. As depicted by FTIR (Fig. 1), ester peak intensity reduced through the addition of bioglass and a new peak assigned to carboxylate was detected $[26,28]$. This is believed to be a result of partial immobilization of polymer chains by increasing the crosslinking density while polymer adsorbed onto the bioglass surface and the physical crosslinks formed [29].

Mechanical properties and porosity

except for POC-BG-30 \%, thermal decomposition occurred through a two-step process. The almost complete weight loss $(99.46 \%)$ was observed for pure POC and thermal decomposition was detected at $650{ }^{\circ} \mathrm{C}$. Similar degradation was observed for all composites between 220 and $600{ }^{\circ} \mathrm{C}$. The thermograms indicated that thermal stability of the scaffolds was enhanced by increasing bioglass content. Composites POC-BG-10 \%, POC-BG-20\%, and POCBG-30 \% demonstrated total weight loss of 88.5, 74 and $71 \%$ respectively. Accordingly, the determined amount of bioglass (TGA) in composites was 11,26 and $28.5 \%$. The comparison between experimental and theoretical values revealed that there is a small discrepancy in glass content

DMA analysis generally provides accurate data for evaluation of the viscoelastic properties of as-prepared scaffolds. The storage modulus $\left(E^{\prime}\right)$, loss modulus $\left(E^{\prime \prime}\right)$, and tan delta $(\tan \delta)$ have been measured at body temperature (Table 1). $T_{\mathrm{g}}$ was determined as the peak of the $\tan \delta$ curve (Fig. 3). Tan $\delta$ represents the ratio of the dissipated energy to the energy stored per cycle $\left(\tan \delta=E^{\prime} / E^{\prime \prime}\right)$ and is often known as damping. Tan $\delta$ value was enhanced by increasing the bioglass content. The storage modulus increased by addition of bioglass in the temperature range of $20-40{ }^{\circ} \mathrm{C}$ and the highest storage modulus was observed for 
Table 1 The thermal and mechanical properties of POCBG scaffolds

Storage modulus, loss modulus and Tan $\delta$ measured for scaffolds at $37^{\circ} \mathrm{C}$

${ }^{\mathrm{a}} T_{\mathrm{g}}=$ Glass transition temperature

${ }^{\mathrm{b}} E_{\mathrm{c}}=$ Compression modulus

\begin{tabular}{lcccc}
\hline Property & POC & POC-BG-10 $\%$ & POC-BG-20 \% & POC-BG-30 \% \\
\hline Storage modulus $(\mathrm{kPa})$ & 25.27 & 42.33 & 49.71 & 81.41 \\
Loss modulus $(\mathrm{kPa})$ & 3.993 & 13.54 & 14.18 & 39.42 \\
Tan $\delta$ at $37{ }^{\circ} \mathrm{C}$ & 0.15 & 0.26 & 0.28 & 0.48 \\
$T_{\mathrm{g}}^{\mathrm{a}}$ at $\tan \delta$ peak $\left({ }^{\circ} \mathrm{C}\right)$ & 0.5 & 4.1 & 9.4 & 12.5 \\
$T_{\mathrm{g}}$ from DSC $\left({ }^{\circ} \mathrm{C}\right)$ & 2.76 & 16.11 & 22.56 & 24.58 \\
$E_{\mathrm{c}}^{\mathrm{b}}(\mathrm{MPa})$ & $0.31 \pm 0.10$ & $2.60 \pm 0.69$ & $4.00 \pm 1.00$ & $6.78 \pm 1.62$ \\
Recovery $(\%)$ & $95.99 \pm 0.72$ & $82.92 \pm 4.65$ & $73.83 \pm 1.60$ & $53.65 \pm 2.58$ \\
Contact angle $\left({ }^{\circ}\right)$ & $61.88 \pm 3.10$ & $59.74 \pm 4.90$ & $60.20 \pm 4.90$ & $58.05 \pm 1.60$ \\
Porosity $(\%)$ & $83.00 \pm 1.87$ & $84.89 \pm 0.68$ & $86.00 \pm 1.01$ & $86.23 \pm 1.73$ \\
\hline
\end{tabular}

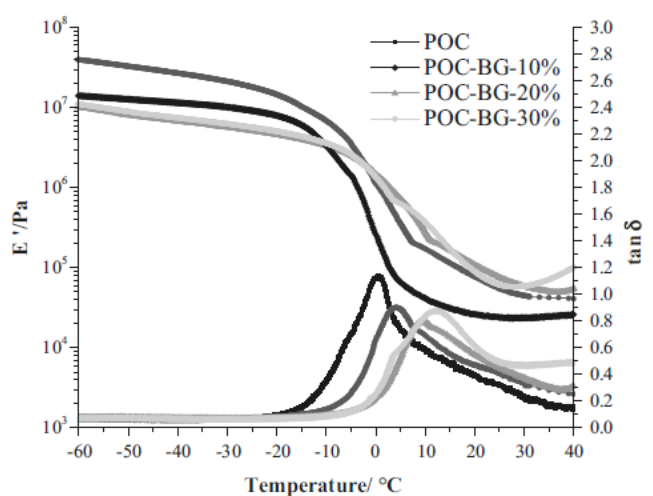

Fig. 3 DMA analysis of composite scaffolds illustrating the typical plots of Tan delta $(\tan \delta)$ and storage modulus $\left(E^{\prime}\right)$ under bending mode

POC-BG-30 \% composite at $81.41 \mathrm{kPa}$. The storage modulus illustrated higher values as compared to loss modulus which confirms the elastic nature of scaffolds. It is believed that addition of bioglass enhances the stiffness since strand density increases due to formation of metallic carboxylate groups [28]. Therefore, the physical interactions between POC and glass improve the storage modulus both below and above $T_{\mathrm{g}}$. The addition of bioglass was seen to increase $T_{\mathrm{g}}$ from $0.5{ }^{\circ} \mathrm{C}$ for pure POC to $12.5^{\circ} \mathrm{C}$ for POC-BG-30 \% composite. All the $T_{\mathrm{g}}$ values obtained by DMA were different from those acquired by DSC (between 2 and $25^{\circ} \mathrm{C}$; Table 1). This may be as a result of partially increased degrees of crosslinking in the first heating step during DSC testing. Moreover, the increase in loss modulus by the addition of bioglass suggests that the composites possess remarkable dissipation capability relative to pure POC [30]. Thus, it can be concluded that addition of bioglass leads to formation of ionic bonds which have significant impact on the partial immobilization of polymer chains.
An ideal material for hard tissue augmentation should have properties that match those of the host bone with suitable load transferrance across the interface to provide mechanical support during bone curing [31]. The incorporation of bioglass particles into the polymer matrix can lead to increasing both strength and load transferral. For this purpose, the prepared scaffolds were tested from a standpoint of compression to investigate the effect of bioglass on mechanical properties. Table 1 summarizes compressive modulus and recovery ratio of the porous scaffolds. The Young's modulus of the scaffolds increased in line with bioglass content and a significant difference was observed between POC-BG-30\% (6.78 MPa) and pure POC (0.31 MPa). However, the recovery ratio has the opposite trend. This can be attributed to enhanced crosslinking density by addition of bioglass which increased the stiffness of the materials [26].

Table 1 also shows measured porosity for composite scaffolds. A scaffold should possess an interconnected and uniformly distributed porosity with a highly porous surface and microstructure [31]. According to previous reported results, a pore size between 200 and $400 \mu \mathrm{m}$ is suitable for cell adhesion and ingrowth in vitro and neovascularization in vivo [4]. When the pores are smaller than this, pore occlusion by the cells could occur, inhibiting ECM production [32]. Prepared scaffolds exhibited high porosities $(>80 \%)$ as displayed in Table 1 . Porosity increased with bioglass concentration; the pure POC scaffold showed the lowest porosity. It is postulated that the addition of bioglass improves structural stability. As a result, POC-BG-30\% had the highest porosity of approximately $86 \%$. This is consistent with the previous study [33] where $20 \%$ incorporation of bioglass into PDLLA led to an increase in porosity and it was more apparent for the nanocomposites in respect to microcomposites [33]. However, the work by Srinivasan et al. showed a decrease in porosity ratio by addition of more nano-bioglass [30]. Overall, the ideal porosity percentage can be defined by the final application 

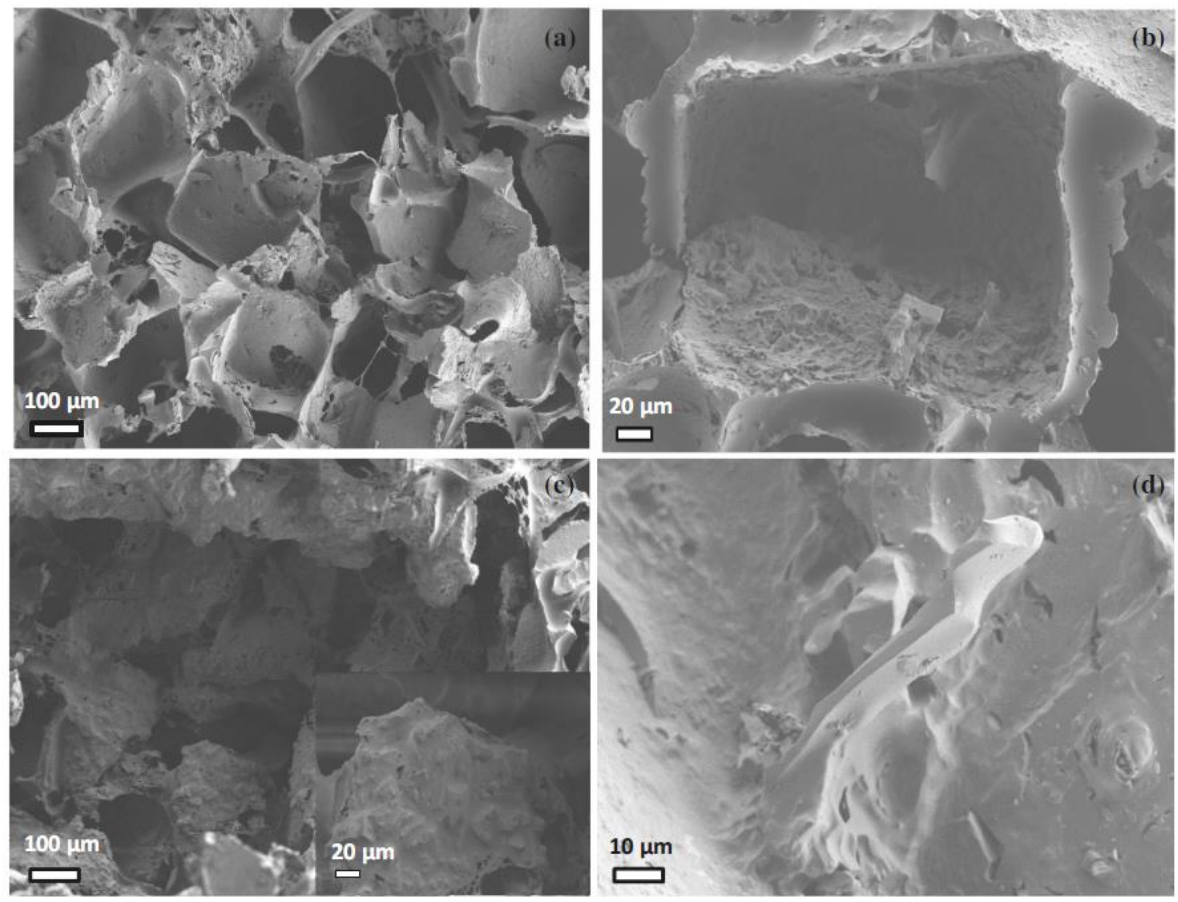

Fig. 4 Microstructure of scaffolds observed by FESEM: a POC; b POC-BG-30 \%; c POC-BG-20 \%; and d POC-BG-10 \%

and also when there is a compromise between mechanical and biological properties [34].

\section{FESEM observation}

Figure 4 shows the FESEM images of both the pure POC and the micro-composites. The images illustrate the porous structure of the scaffolds (Fig. 4a) with pore size recorded in the range of 200-300 $\mu \mathrm{m}$ (Fig. 4b). All the scaffolds showed consistent porous structures as detected by SEM. In addition, it can be seen that bioglass particles are well-dispersed and embedded into the polymer matrix (Fig. 4c) which in turn improve the load transferral across the filler-matrix interface and amend the composite stiffness [35]. However, the larger particles were not fully incorporated into the matrix (Fig. 4d) and can be easily debonded from the POC. Therefore, the particles cannot transfer any loads which consequently lead to reducing composite strength [36].

In vitro degradation study

The degradation of pure POC and composite scaffolds was investigated in PBS. Figure 5 shows the weight loss of all

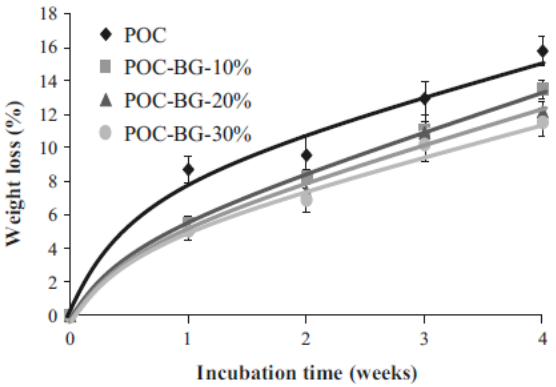

Fig. 5 In vitro degradation profile of composite scaffolds (PBS; $\left.37^{\circ} \mathrm{C}\right)$

prepared scaffolds. The data revealed that increasing the concentration of bioglass reduced the degradation rate as reported in terms of weight loss. Pure POC completely degraded after 6 months [18]. In our case, the pure POC scaffold had the highest percentage of weight loss at about $16 \%$ during 4 weeks of incubation and POC-BG-30\% demonstrated the lowest weight loss which is probably due 

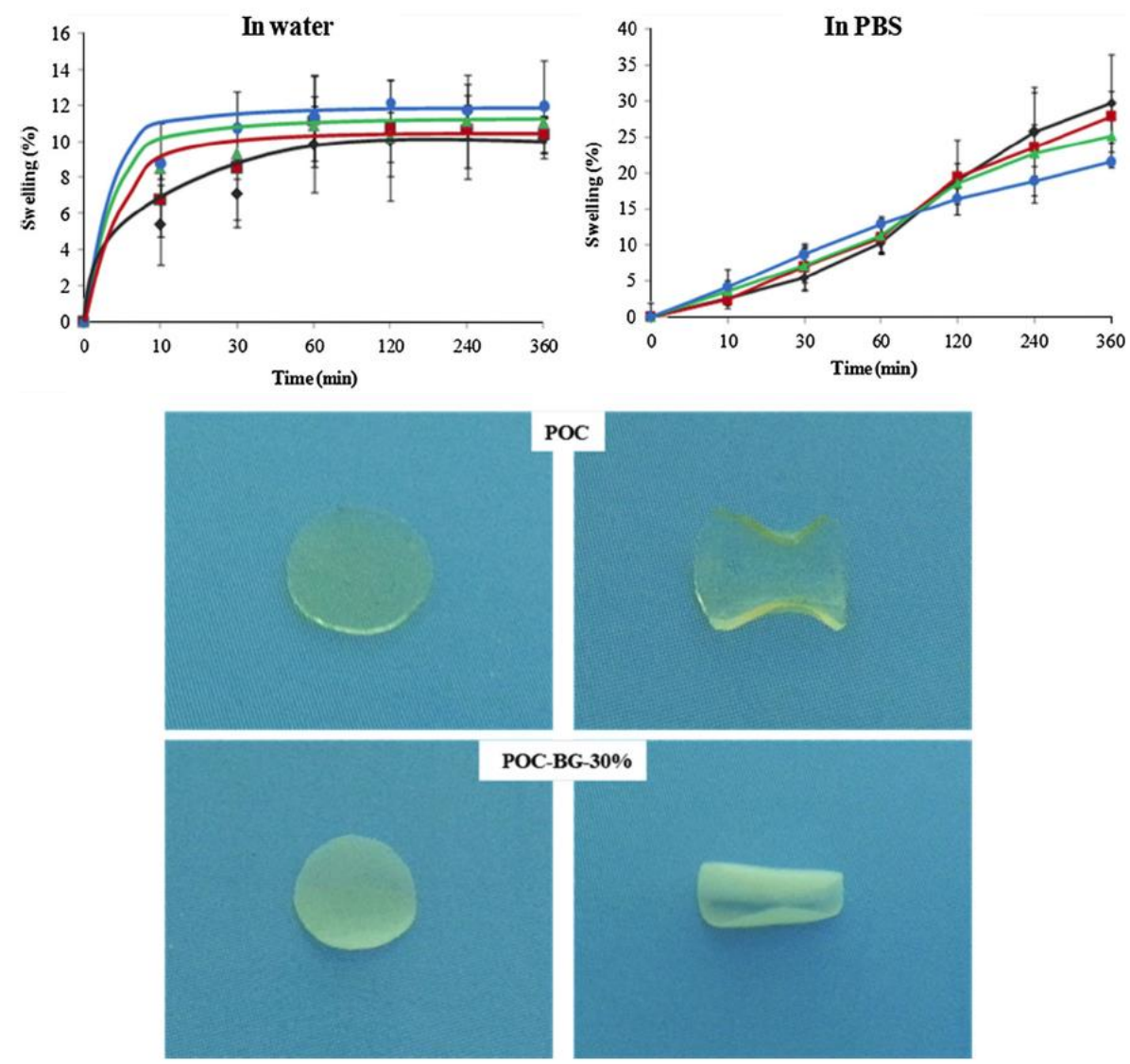

OC-BG-30\%

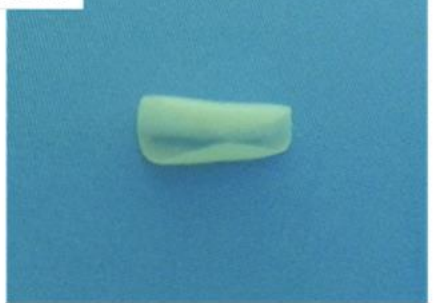

Fig. 6 Swelling ratio in water and PBS of: pure POC (black-filled diamond); POC-BG-10\% (red-filled square); POC-BG-20\% (greenfilled triangle); and POC-BG-30\% (blue-filled circle). The images

show films of POC and POC-BG-30 \% after soaking in water (left) and PBS (right) for $4 \mathrm{~h}$ (Color figure online)

to the slow dissolution of bioglass. The low dissolution of bioglass is assessed by enhancing the acidic nature of the bioglass surface as a result of the presence of $\mathrm{Ga}$ [37]. Liang et al. ascribed the low degradation of composites from poly(glycerol sebacate) (PGS) and 45S5 bioglass to the variation in $\mathrm{pH}$ as bioglass has ability to release the alkaline ions and keep the buffer solution neutralized [38]. Our composite scaffolds showed similar behavior. The $\mathrm{pH}$ study in PBS demonstrated the $\mathrm{pH}$ variation was not significantly influenced by the POC-BG-30 \% and it almost kept constant around 7.2. All the scaffolds illustrated a decrease in $\mathrm{pH}$ at day 1 from 7.4 (PBS) to 6.93, 7.04, 7.12, and 7.16 for pure POC, POC-BG-10 \%, POC-BG- $20 \%$, and POC-BG-30 \%, respectively (data not shown). After the initial drop the $\mathrm{pH}$ values remained almost constant over the course of 7 days.

Water in air contact angle

Wettability plays a crucial role at the interface of a biomaterial with a biological system. Different biological events such as cell attachment, cell proliferation and protein adsorption can be considerably affected by wettability at the interface [39]. Static contact angle measurements were carried out to measure the hydrophilicity of the prepared films and the data are displayed in Table 1. There were small differences between the wettability of all the compositions and the results were in the range of $58^{\circ}-62^{\circ}$. Alhalawani et al. reported that the addition of $\mathrm{Ga}$ to a bioglass led to increased hydrophilicity which in turn can be a reason for enhancement of wettability of our composites [40]. The composite films indicated lower contact

\section{Link to Full-Text Articles :}

http://link.springer.com/article/10.1007/s10853-014-8782-2 